\title{
Radiation dose estimates in dual-source computed tomography coronary angiography
}

\author{
Stolzmann, P ; Scheffel, H ; Schertler, T ; Frauenfelder, T ; Leschka, S ; Husmann, L ; Flohr, T G ;
} Marincek, B ; Kaufmann, P A ; Alkadhi, H

\begin{abstract}
The purpose of this study was to quantify radiation dose parameters of dual-source CT coronary angiography. Eighty patients underwent contrast-enhanced, retrospectively ECG-gated dual-source CT coronary angiography with heart rate-adapted ECG pulsing using two algorithms: In 40 patients, the tube current was reduced to $20 \%(\mathrm{~A}(\min 1))$ of the normal tube current $(\mathrm{A}(\max ))$ outside the pulsing window; in 40 patients tube current was reduced to $4 \%(\mathrm{~A}(\min 2))$ of $\mathrm{A}(\max )$. Mean $\mathrm{CTDI}(\mathrm{vol})$ in the $\mathrm{A}(\mathrm{min} 1)$ group was $45.1+/-3.6 \mathrm{mGy}$; the mean $\mathrm{CTDI}(\mathrm{vol})$ in the $\mathrm{A}(\min 2)$ group was $39.1+/-3.2 \mathrm{mGy}$, with $\mathrm{CTDI}(\mathrm{vol})$ in the $\mathrm{A}(\mathrm{min} 2)$ group being significantly reduced when compared to the $\mathrm{A}(\mathrm{min} 1)$ group $(\mathrm{P}<0.001)$. A significant negative correlation was found between CTDI(vol) and heart rate in group $\mathrm{A}(\min 1)(\mathrm{r}=-0.82, \mathrm{P}<0.001)$, whereas no correlation was found between $\mathrm{CTDI}(\mathrm{vol})$ and heart rate in group $\mathrm{A}(\min 2)(\mathrm{r}=-0.066)$. Using the conversion coefficient for the chest, dual-source CT coronary angiography resulted in an estimated mean effective dose of $8.8 \mathrm{mSv}$ in the $\mathrm{A}(\mathrm{min} 1)$ group and $7.8 \mathrm{mSv}$ in the A(min2). Radiation exposure of dual-source CT coronary angiography using an ECG-pulsing protocol reducing the tube current to $20 \%$ significantly decreases with increasing heart rates, despite using wider pulsing windows at higher heart rates. When using a protocol with reduced tube current of $4 \%$, the radiation dose is significantly lower, irrespective of the heart rate.
\end{abstract}

DOI: https://doi.org/10.1007/s00330-007-0786-8

Posted at the Zurich Open Repository and Archive, University of Zurich

ZORA URL: https://doi.org/10.5167/uzh-3611

Journal Article

Published Version

Originally published at:

Stolzmann, P; Scheffel, H; Schertler, T; Frauenfelder, T; Leschka, S; Husmann, L; Flohr, T G; Marincek, B; Kaufmann, P A; Alkadhi, H (2008). Radiation dose estimates in dual-source computed tomography coronary angiography. European Radiology, 18(3):592-599.

DOI: https://doi.org/10.1007/s00330-007-0786-8 
Paul Stolzmann

Hans Scheffel

Thomas Schertler

Thomas Frauenfelder

Sebastian Leschka

Lars Husmann

Thomas G. Flohr

Borut Marincek

Philipp A. Kaufmann

Hatem Alkadhi

\section{Radiation dose estimates in dual-source computed tomography coronary angiography}

Received: 18 July 2007

Revised: 15 August 2007

Accepted: 11 September 2007

Published online: 2 October 2007

(C) European Society of Radiology 2007

P. Stolzmann - H. Scheffel .

T. Schertler · T. Frauenfelder

S. Leschka $\cdot$ L. Husmann ·

B. Marincek · H. Alkadhi $(\square)$

Institute of Diagnostic Radiology,

University Hospital Zurich,

Raemistrasse 100,

8091 Zurich, Switzerland

e-mail: hatem.alkadhi@usz.ch

Tel.: +41-44-2553662

Fax: +41-44-2554443

T. G. Flohr

Siemens Medical Solutions,

Forchheim, Germany

P. A. Kaufmann

Cardiovascular Center,

Forchheim, Germany
Abstract The purpose of this study was to quantify radiation dose parameters of dual-source CT coronary angiography. Eighty patients underwent contrast-enhanced, retrospectively ECG-gated dual-source CT coronary angiography with heart rate-adapted ECG pulsing using two algorithms: In 40 patients, the tube current was reduced to $20 \%\left(\mathrm{~A}_{\min 1}\right)$ of the normal tube current $\left(\mathrm{A}_{\max }\right)$ outside the pulsing window; in 40 patients tube current was reduced to $4 \%\left(\mathrm{~A}_{\min 2}\right)$ of $A_{\max }$. Mean $C$ TDI $_{\mathrm{vol}}$ in the $\mathrm{A}_{\min 1}$ group was $45.1 \pm 3.6 \mathrm{mGy}$; the mean $\mathrm{CTDI}_{\mathrm{vol}}$ in the $\mathrm{A}_{\min 2}$ group was $39.1 \pm$ $3.2 \mathrm{mGy}$, with $\mathrm{CTDI}_{\mathrm{vol}}$ in the $\mathrm{A}_{\min 2}$ group being significantly reduced when compared to the $\mathrm{A}_{\min 1}$ group $(\mathrm{P}<$ $0.001)$. A significant negative correlation was found between $\mathrm{CTDI}_{\mathrm{vol}}$ and heart rate in group $\mathrm{A}_{\min 1}(\mathrm{r}=-0.82, \mathrm{P}<$
0.001 ), whereas no correlation was found between $\mathrm{CTDI}_{\mathrm{vol}}$ and heart rate in group $\mathrm{A}_{\min 2}(\mathrm{r}=-0.066)$. Using the conversion coefficient for the chest, dual-source CT coronary angiography resulted in an estimated mean effective dose of $8.8 \mathrm{mSv}$ in the $\mathrm{A}_{\min 1}$ group and $7.8 \mathrm{mSv}$ in the $\mathrm{A}_{\min 2}$. Radiation exposure of dual-source CT coronary angiography using an ECG-pulsing protocol reducing the tube current to $20 \%$ significantly decreases with increasing heart rates, despite using wider pulsing windows at higher heart rates. When using a protocol with reduced tube current of $4 \%$, the radiation dose is significantly lower, irrespective of the heart rate.

Keywords Coronary angiography . Dual-source computed tomography . Radiation dose

\section{Introduction}

Non-invasive coronary angiography using multi-slice computed tomography (CT) has recently emerged as an important clinical tool for the accurate assessment of coronary artery disease (CAD) [1-4]. Lately introduced dual-source CT scanners are characterized by two X-ray tubes and two corresponding detector units that are perpendicularly mounted onto the rotating gantry. This scanner provides a temporal resolution of $83 \mathrm{~ms}$ in a mono-segment reconstruction mode [5]. Owing to the improved temporal resolution, cardiac CT examinations with dual-source CT have been shown to provide diagnostic image quality even at higher heart rates [6-8] that potentially will increase the use of CT for diagnostic workup of the coronary arteries.
CT represents the most important source of ionizing radiation arising from medical exposures [9]. As the basic principle of radiation protection, harmful radiation exposure should be kept "as low as reasonably achievable (ALARA)." This principle must be kept in mind particularly regarding electrocardiography (ECG)-gated CT coronary angiographies that are dose-intensive examinations $[10,11]$. For reducing radiation dose in cardiac CT, the technique of ECG-based tube current modulation has been introduced, which is characterized by modulation of the tube current throughout the cardiac cycle allowing reduction of the dose by as much as 50\% [12]. Dual-source CT scanners allow for flexible ECG-based tube current modulation with variable window widths of the full current phases that can be narrowed down to a temporal window of 
$110 \mathrm{~ms}$ [5]. In addition, radiation dose is further minimized through adaptation of the pitch to the heart rate, with an increase in pitch at higher heart rates [13]. This adaptation directly leads to a decrease in patient dose, as the average dose within the scan volume is directly proportional to $1 /$ pitch [11].

McCollough and coworkers have recently shown that the simultaneous operation of two X-ray tubes need not contribute to an increase in radiation dose for ECG-gated cardiac CT examinations [13]. In a phantom study, the authors have demonstrated a reduction in dose by a factor of up to two with equivalent image noise relative to singlesource CT, depending on the heart rate. However, this phantom study made assumptions about the appropriate temporal window width by choosing a window of $110 \mathrm{~ms}$ at lower heart rates and a wider window of $330 \mathrm{~ms}$ at higher heart rates that may not be adequate in patients [13].

The aim of this study was to quantify radiation dose estimates in patients undergoing dual-source CT coronary angiography.

\section{Materials and methods}

Patients

Eighty patients (32 females, 48 males, mean age $65 \pm$ 11 years, range $45-87$ years) who have had a clinically indicated CT coronary angiography examination were retrospectively included in this study. Exclusion criteria for CT coronary angiography were renal insufficiency (serum creatinine level $>150 \mu \mathrm{mol} / \mathrm{l}$ ) and allergy to iodinated contrast media. Reasons for referral were atypical chest pain in combination with a low- to intermediate pre-test probability of coronary artery disease in all patients. The local ethical committee approved the study; written informed consent was waived.

\section{Dual-source CT scanning protocol}

Three minutes prior to the scan, patients received a single dose of $2.5 \mathrm{mg}$ s.l. isosorbiddinitrate (Isoket, Schwarz Pharma, Monheim, Germany). No beta receptor antagonists for heart rate control were administered even in patients with high or irregular heart rates.

All CT examinations were performed on a dual-source CT scanner (Somatom Definition, Siemens Medical Solutions, Forchheim, Germany). Contrast-enhanced dual-source CT covered a scan range from a level of the aortic root to the diaphragm. A biphasic contrast media protocol was administered that was adjusted to the varying scan duration. A bolus of $60-80 \mathrm{ml}$ contrast media (Visipaque $320,320 \mathrm{mg} / \mathrm{mL}$, GE Healthcare, Buckinghamshire, UK) was injected into an antecubital vein via an 18 -gauge catheter with a flow rate of
$5 \mathrm{ml} / \mathrm{s}$ followed by the same amount as the first phase with a dilution of 1:5 parts saline solution.

Contrast agent application was controlled by bolus tracking. The region of interest was placed into the aortic root, and image acquisition started $5 \mathrm{~s}$ after the signal attenuation reached the predefined threshold of 140 Hounsfield units (HU). Data acquisition was performed in a cranio-caudal direction with a tube current-time product of $350 \mathrm{mAs}$ per rotation, tube potential of $120 \mathrm{kV}$, detector collimation of $2 \times 32 \times 0.6 \mathrm{~mm}$, slice acquisition of $2 \times 64 \times 0.6 \mathrm{~mm}$ by means of a z-flying focal spot, gantry rotation time $330 \mathrm{~ms}$, and pitch of $0.2-0.5$ automatically adapted to the heart rate.

\section{ECG-gated pulsing}

ECG pulsing of tube current for radiation dose reduction was used in all 80 patients (Fig. 1). The pulsing window (in \% of the RR interval) was adjusted to the individual heart rate of the patient as previously recommended [14] (Fig. 2).

\section{At heart rates}

1. below 60 beats per minute (bpm), full tube current $\left(A_{\max }\right)$ was applied from 60 to $70 \%$,

2. from 60 to $69 \mathrm{bpm}, \mathrm{A}_{\max }$ was applied from 60 to $80 \%$,

3. from 70 to $79 \mathrm{bpm}, \mathrm{A}_{\max }$ was applied from 55 to $80 \%$, and

4. above $79 \mathrm{bpm}, \mathrm{A}_{\max }$ was applied from 30 to $80 \%$.

The full tube current $\left(\mathrm{A}_{\max }\right)$ was reduced outside the adjusted pulsing windows

1. to a minimum tube current $A_{\min 1}=0.20 \times A_{\max }$ in 40 patients and

2. to a minimum tube current $A_{\min 2}=0.04 \times A_{\max }$ (MinDose, Siemens) in 40 patients.

\section{Dual-source CT image reconstruction}

Retrospective ECG-gating pulsing was used to synchronize the data with the ECG. A mono-segment reconstruction algorithm was used [5]. Images were reconstructed with a slice thickness of $0.75 \mathrm{~mm}$ and an increment of $0.4 \mathrm{~mm}$ using a soft-tissue convolution kernel (B26f). Images were reconstructed in $5 \%$ steps of the RR-interval within the window of full tube current. All data were transferred to an evaluation workstation (Siemens).

\section{Estimation of radiation dose}

The effective dose of CT coronary angiography was estimated by a method proposed by the European Working Group for Guidelines on Quality Criteria in CT [15]. This method has been shown to be of reasonable robustness and 
Fig. 1 Triage of the 80 patients (n: number of patients per group). In 40 patients the minimum tube current $\left(\mathrm{A}_{\min 1}\right)$ was $20 \%$ of the normal tube current $\left(\mathrm{A}_{\max }\right)$ outside the described pulsing window. In 40 patients, the minimum tube current $\left(A_{\min 2}\right)$ was reduced to $4 \%$ of the full tube current $\left(\mathrm{A}_{\max }\right)$ outside the pulsing window. The normal tube current $\left(\mathrm{A}_{\max }=\right.$ $100 \%$ ) was applied at different pulsing window widths adjusted to the individual patient's heart rate

Fig. 2 a Illustration of the two ECG-based tube current modulation algorithms used in the study. In group $\mathrm{A}_{\min 1}$ the normal tube current was reduced to $20 \%$; in group $A_{\min 2}$ the full tube current was reduced to $4 \%$ of $A_{\max }$ outside the window of normal tube current. Note the different amount of image noise in the three representative images with $100 \%, 20 \%$, and $4 \%$ of the normal tube current. Whereas analysis of ventricular function is still possible using images at $20 \%$ tube current, images at $4 \%$ can no longer be used for this purpose. b Oblique multi-planar reformations of the proximal left coronary artery showing the different image noise with $100 \%, 20 \%$, and $4 \%$ of the normal tube current

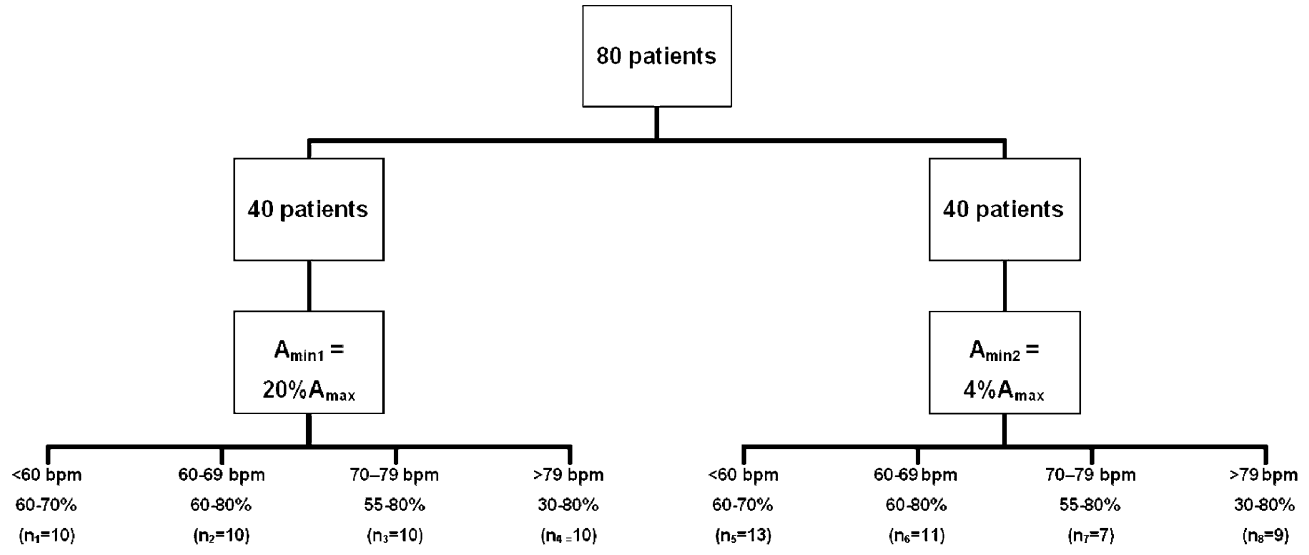

a

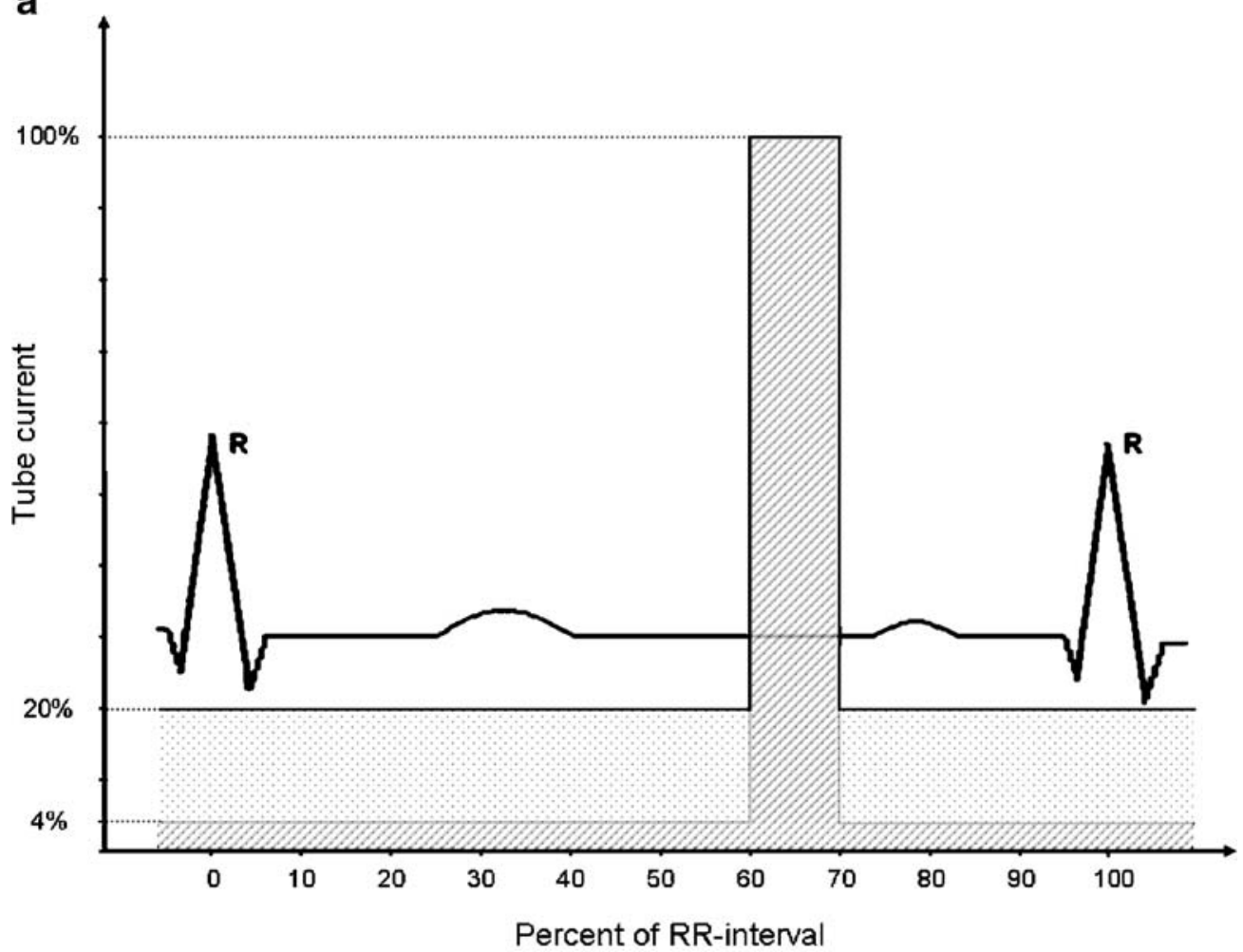

b

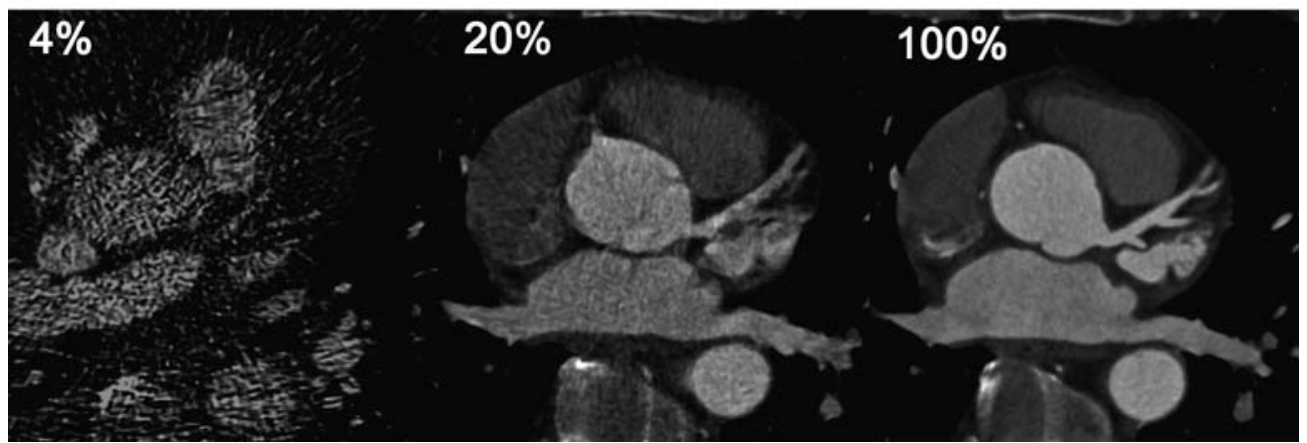


high consistency for estimating the effective dose [16]. The effective dose was derived from the product of the doselength product and a conversion coefficient for the chest as the investigated anatomic region. This conversion coefficient $(\mathrm{k}=0.017 \mathrm{mSv} /(\mathrm{mGy} \times \mathrm{cm}))$ is averaged between male and female models from Monte Carlo simulations [17]. This technique is used to determine specific organ doses by simulating the absorption and scattering of X-ray photons in various tissues by using a mathematical model of the human body.

The parameters for the dose-length product and $\mathrm{CTDI}_{\mathrm{vol}}$, which averages the radiation dose in the $\mathrm{x}-, \mathrm{y}-$ and $\mathrm{z}-$ directions, were obtained from the protocol that summarized the relevant individual radiation exposure parameters for the coronary CT investigation. The scan length (z) and pitch of the CT coronary angiography examinations were recorded in all patients.

Conventional CT dose metrics are fully applicable to dual-source CT because the radiation from each tube add together in a straightforward linear manner, with each dose independent of the other [13]. Hence, measurements with each tube separately energized or with both tubes simultaneously energized result in the same combined dose values.

\section{Image quality}

To assess the impact of the two different scanning protocols on image quality, a qualitative assessment of coronary segments was performed. For data analysis, coronary segments were determined according to recommendations of the American Heart Association from 1975 [18]. All reconstructed images were evaluated and classified by two independent and blinded readers (with 2 and 9 years of experience in cardiovascular radiology) using transverse source images, multi-planar reformations (MPR), and thinslab maximum intensity projections (MIP) on a per-segment basis.

Both readers assessed the image quality of each coronary segment on a 2-point ranking scale:

1. Excellent (no artifacts), good (mild artifacts, unrestricted evaluation), or adequate (moderate artifacts, but still diagnostic) image quality, and

2. Poor image quality (severe artifacts leading to nondiagnostic examinations) due to motion artifacts (defined as vessel movement resulting in blurred or doubled vessel contours).

For any disagreement in data analysis, consensus agreement was achieved.

\section{Statistical analysis}

Numerical values of continuous variables are expressed as means \pm standard deviations (SD) and categorical var- iables as frequencies or percentages. For ordinal variables, comparisons of the two groups were performed with the chi-square test and Fisher's exact test, where appropriate. For continuous data, correlation analysis was performed using Spearman rank order correlation coefficients. MannWhitney-U tests were used to ascertain the significance of differences. A $P$-value of less than 0.05 was considered to indicate statistical significance. Data analysis was performed using commercially available software (SPSS 12.0, Chicago, IL).

\section{Results}

Dose parameters $\left(\mathrm{CTDI}_{\mathrm{vol}}, \mathrm{DLP}\right.$, and $\left.\mathrm{z}\right)$ were available in the patient protocols of all examinations. The mean scan range (z) was $124 \pm 11 \mathrm{~mm}$ (range $97-147 \mathrm{~mm}$ ). All patients were in sinus rhythm with a mean heart rate of $70 \pm 17 \mathrm{bpm}$ (range 36-124 bpm), no premature heart beats occurred. ECG-based tube current modulation was successfully applied in all examinations.

In group $\mathrm{A}_{\min 1}$ (mean heart rate $72 \pm 18 \mathrm{bpm}$, range 50 $124 \mathrm{bpm}$ ), ten patients had a heart rate below $60 \mathrm{bpm}$ (mean $54 \pm 3 \mathrm{bpm}$, range 50-59 bpm) with $\mathrm{A}_{\max }$ applied from 60 $70 \%$ of the RR interval; ten patients had a heart rate between 60-69 bpm (mean 64 $\pm 2 \mathrm{bpm}$, range 61-68 bpm) with $\mathrm{A}_{\max }$ applied from $60-80 \%$ of the RR-interval; ten patients had a heart rate between 70-79 bpm (mean 74 $3 \mathrm{bpm}$, range $71-79 \mathrm{bpm}$ ) with $\mathrm{A}_{\max }$ applied from 55-80\% of the RR interval, ten patients had a heart rate above $79 \mathrm{bpm}$ (mean $97 \pm 14 \mathrm{bpm}$, range 82-124 bpm) with $\mathrm{A}_{\max }$ applied from $55-80 \%$ of the RR interval (see Fig. 1).

In group $\mathrm{A}_{\min 2}$ (mean heart rate $67 \pm 15 \mathrm{bpm}$, range 36$100 \mathrm{bpm}$ ), 13 patients had a heart rate below $60 \mathrm{bpm}$ (mean $51 \pm 7 \mathrm{bpm}$, range $36-59 \mathrm{bpm}$ ) with $A_{\max }$ from $60-70 \%$ of the RR-interval; 11 patients had a heart rate between 60 $69 \mathrm{bpm}$ (mean $63 \pm 3 \mathrm{bpm}$, range 60-68 bpm) with $A_{\max }$ was applied from $60-80 \%$ of the RR-interval; 7 patients had a heart rate between 70-79 bpm (mean 76 $\pm 2 \mathrm{bpm}$, range $74-79 \mathrm{bpm}$ ) with $\mathrm{A}_{\max }$ from $55-80 \%$ of the RRinterval; 9 patients had a heart rate above $79 \mathrm{bpm}$ (mean $89 \pm 7 \mathrm{bpm}$, range $82-100 \mathrm{bpm}$ ) with $\mathrm{A}_{\max }$ from $55-80 \%$ of the RR interval (see Fig. 1).

No significant differences were found regarding heart rate $(\mathrm{P}=$ n.s. $)$ and scan range $(\mathrm{P}=$ n.s. $)$ between group $\mathrm{A}_{\min 1}$ and group $\mathrm{A}_{\min 2}$.

\section{Estimation of radiation dose}

In group $\mathrm{A}_{\min 1}$, mean $\mathrm{CTDI}_{\mathrm{vol}}$ was $45.1 \pm 3.6 \mathrm{mGy}$ (range 39.6-54.5 mGy) and mean DLP was $522.1 \pm 40.9 \mathrm{mGy} \times$ $\mathrm{cm}$ (range 537.2-592.8 $\mathrm{mGy}$ ). Calculation of the effective dose (product of DLP with the conversion coefficient for the chest) revealed a mean Dose $_{\text {eff }}$ of $8.8 \pm 0.7 \mathrm{mSv}$ (Table 1). 
Table 1 Dose estimates of dual-source CT coronary angiography using ECG-based tube current modulation in two groups with different tube current reduction outside the pulsing windows

\begin{tabular}{llll}
\hline & CTDI $_{\text {vol }}[\mathrm{mGy}]$ & DLP [mGy cm] & Dose $_{\text {eff }}[\mathrm{mSv}]$ \\
\hline $\mathrm{A}_{\min 1}(\mathrm{n}=40)$ & $45.1 \pm 3.6$ & $522.1 \pm 40.9$ & $8.8 \pm 0.7$ \\
$\mathrm{~A}_{\min 2}(\mathrm{n}=40)$ & $39.1 \pm 3.2$ & $459.4 \pm 62.7$ & $7.8 \pm 1.1$ \\
\hline
\end{tabular}

$A_{\min 1}$ : tube current reduction to $20 \%$ of the full tube current $\left(A_{\max }\right)$, $A_{\min 2}$ : tube current reduction to $4 \%$ of the full tube current $\left(A_{\max }\right)$, $\mathrm{CTDI}_{\mathrm{vol}}$ : computed tomography dose index, DLP: dose length product, Dose $_{\text {eff: }}$ effective Dose

In group $\mathrm{A}_{\text {min2 } 2}$, mean $\mathrm{CTDI}_{\mathrm{vol}}$ was $39.1 \pm 3.2 \mathrm{mGy}$ (range 31.9-46.8 $\mathrm{mGy}$ ) and mean DLP was $459.4 \pm$ $62.7 \mathrm{mGy} \times \mathrm{cm}$ (range 338.2-555.0 $\mathrm{mGy}$ ). Calculation of the effective dose revealed a mean Dose $_{\text {eff }}$ of $7.8 \pm 1.1 \mathrm{mSv}$ (Table 1).

Significant differences were found between group $A_{\min 1}$ and group $\mathrm{A}_{\min 2}$ for $\mathrm{CTDI}_{\mathrm{vol}}(\mathrm{P}<0.001)$, DLP $(\mathrm{P}<0.001)$, and $\operatorname{Dose}_{\text {eff }}(\mathrm{P}<0.001)$.

A significant negative correlation was found between $\mathrm{CTDI}_{\mathrm{vol}}$ and heart rate in group $\mathrm{A}_{\min 1}\left(r_{\mathrm{Amin} 1}=-0.82, \mathrm{P}<\right.$ 0.001) (Fig. 3a). No significant correlation was found between $\mathrm{CTDI}_{\mathrm{vol}}$ and heart rate in group $\mathrm{A}_{\min 2}\left(r_{\mathrm{Amin} 2}=\right.$ $-0.066, \mathrm{P}=$ n.s.) (Fig. 3b).

Image quality

A total of 116 segments was missing because of anatomical variants (primarily due to presence or absence of segments 15 and 16). A total of 1,164 coronary segments was evaluated regarding image quality.

Inter-observer agreement for image quality rating was excellent $(\mathrm{kappa}=0.81)$. Immediate agreement between both observers was achieved in $98 \%$ of the segments, while consensus reading was necessary in the remaining $2 \%$. Image quality was considered by both readers as being diagnostic (score 1) in 1,129/1,164 (97\%) of the segments and as being non-diagnostic (score 2) in 35/1,164 (3\%) of the segments. Reasons for non-diagnostic image quality were extensive motion artifacts in all. Coronary segments with non-diagnostic image quality were all distal side branches (segments 4, 10, 14 , and 15). No significant differences $(\mathrm{P}=$ n.s. $)$ regarding image quality were found comparing that of protocol $\mathrm{A}_{\min 1}$ and protocol $\mathrm{A}_{\min 2}$.

\section{Discussion}

This study demonstrates that dual-source CT coronary angiography using a protocol with $350 \mathrm{mAs} /$ rotation and $120 \mathrm{kV}$ results in an average estimated radiation dose of approximately $8.8 \mathrm{mSv}$ when implementing an ECGpulsing with tube current reduction to $20 \%$. With this protocol, radiation dose significantly decreases at increasing heart rates. Using the same tube current and voltage and an ECG-pulsing with tube current reduction to 4\%, average estimated radiation dose is significantly lower being approximately $7.8 \mathrm{mSv}$ and stays relatively constant across different heart rates.

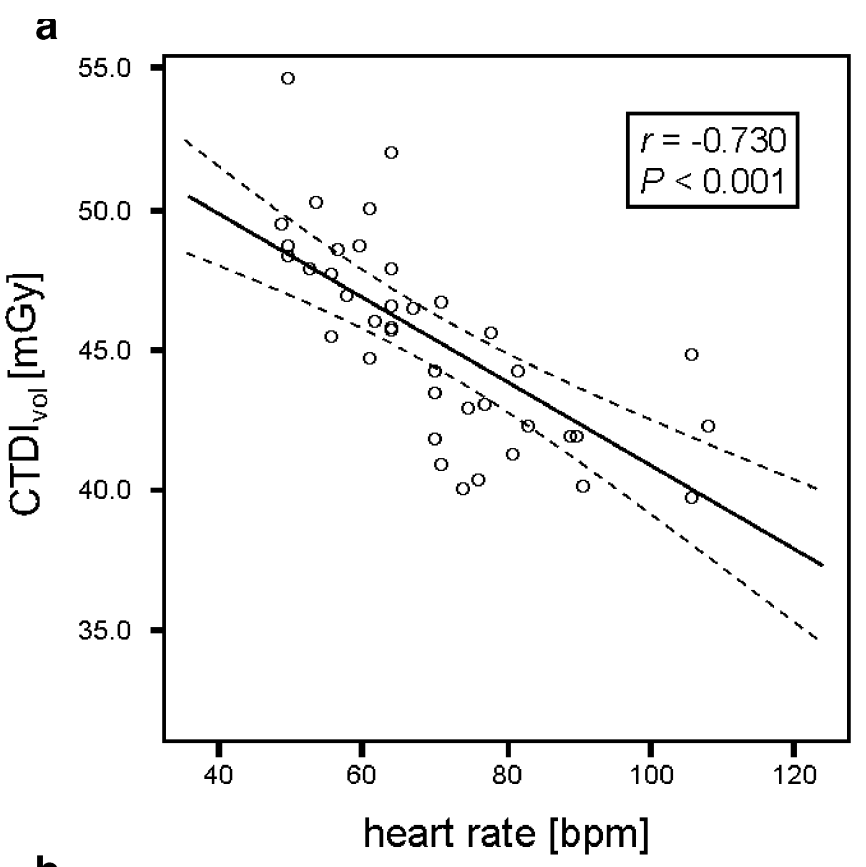

b

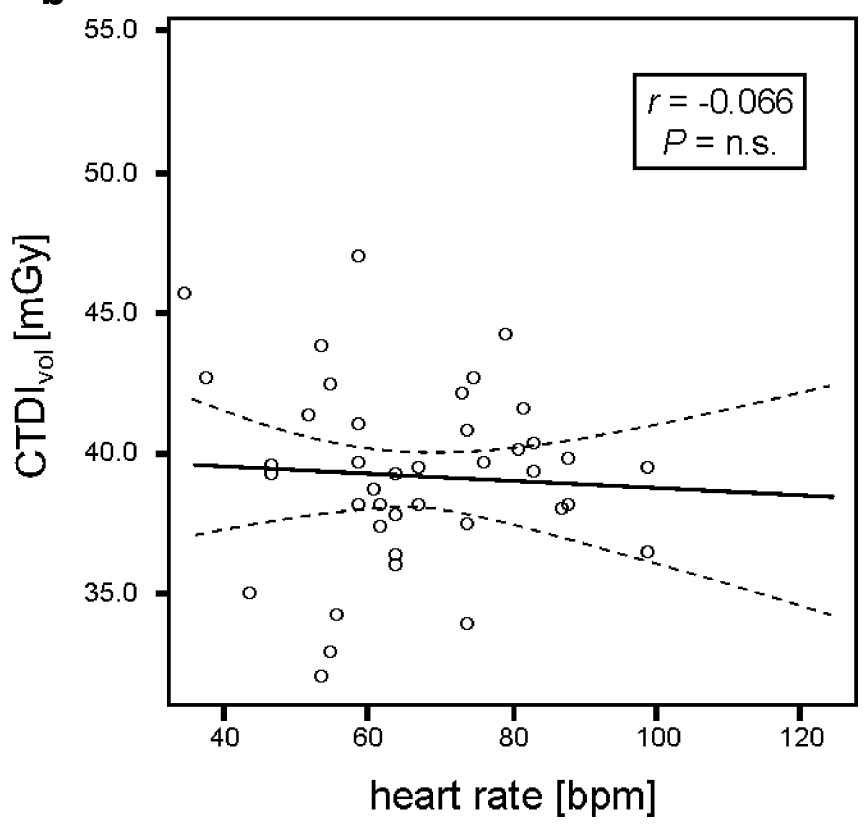

Fig. 3 Correlation between $\mathrm{CTDI}_{\mathrm{vol}}[\mathrm{mGy}]$ and heart rate $[\mathrm{bpm}]$ in the two patient groups. (a) A significant negative correlation was found for group $\mathrm{A}_{\min 1}(\mathrm{r}=-0.730, \mathrm{P}<0.001)$. (b) No significant correlation was found for group $\mathrm{A}_{\min 2}(\mathrm{r}=-0.066, \mathrm{P}=\mathrm{n} . \mathrm{s}$.) 


\section{CT dose parameters}

In order to describe medical radiation exposure, different dose parameters are in use. Most useful parameters to express and compare different scanning protocols are $\mathrm{CTDI}_{\mathrm{vol}}$, DLP, and Dose eff $_{\text {[10]. }}$.

The $\mathrm{CTDI}_{\mathrm{vol}}$ takes the average radiation dose in all three directions (i.e., $\mathrm{x}-, \mathrm{y}$-, and $\mathrm{z}$-direction) into account. It provides an easily measurable dose parameter, which estimates the average radiation within the irradiated volume of one CT acquisition based on a standardized phantom [19]. The value of the $\mathrm{CTDI}_{\mathrm{vol}}$ is directly related to the overlap between adjacent slices, which varies by the slice thickness and the distance between the acquired slices. Distance increases in a helical scanning mode by an increasing pitch. The DLP reflects the total energy absorbed by the patient that represents the $\mathrm{z}$-directional integration of the $\mathrm{CTDI}_{\mathrm{vol}}[10]$. Therefore, the scan length has to be kept as short as possible. In order to describe the risk of a partial, non-uniform body exposure relative to an equivalent whole-

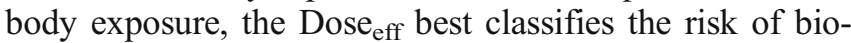
logical injuries [20].

\section{Radiation dose in cardiac CT}

In cardiac single-source multi-slice $\mathrm{CT}$, faster rotation times deliver an improved temporal resolution, but do require a slower pitch to avoid discontinuities of the anatomic coverage when images are reconstructed from consecutive cardiac cycles [11]. Hence for most single-source multidetector row CT systems, better temporal resolution in cardiac spiral CT comes with the price of a higher dose [11]. These conclusions do not apply to the dual-source CT system [13], which allows heart rate-adapted pitch values and a temporal resolution of $83 \mathrm{~ms}$ providing singlesegment reconstructions at all heart rates [5]. Therefore, the pitch can be increased at elevated heart rates because multisegment reconstruction must not be used [5]. The higher table travel velocity effectuates the duration of exposure and thereby reduces dose, not only by reducing the overlap of adjacent slices [5]. In a recent phantom study evaluating the dose performance of dual source CT, the most severe dose reductions were shown as the heart rate increased and therefore the pitch raised [13].

We found a significant reduction of $\mathrm{CTDI}_{\mathrm{vol}}$ with higher heart rates using the ECG-based tube current modulation with lowering of the tube current to $20 \%$ outside the normal tube output window. Importantly, this reduction was present despite continuously wider ECG-pulsing windows from $60-70 \%$ at lower to $30-80 \%$ of the R-R interval at higher heart rates [14]. This confirms the predominant role of increasing pitch for radiation dose reduction that outweighs the wider window with full tube current at higher heart rates.
In contrast, increasing the pitch at higher heart rates did not result in decreasing radiation when using the ECGbased tube current modulation protocol with reduction to $4 \%$ outside the full tube current window. With this protocol, dose stayed relatively constant at a lower level. Comparing the two scatterplots in Fig. 3 shows that radiation dose saving using the ECG-pulsing protocol with $4 \%$ reduction is most effective at lower heart rates.

Compared to previous 64-slice CT scanners, the generators and rotating envelope tubes used in dual-source CT systems allow faster tube current transitions from full to low tube current and vice versa [21]. This results in a decrease of total dose delivered to obtain the maximum tube current for the desired ECG-pulsing window, as less time is spent ramping up to and down from the target tube current [13]. With increasing heart rates, the window for tube current reduction gets shorter, both due to wider pulsing windows and shorter absolute R-R-interval duration. The most likely explanation for constant radiation at high heart rates with the $4 \%$-tube current reduction protocol is that the time to ramp-down and ramp-up the tube may be still too long. The other way around, at lower heart rates, tube current reduction to $4 \%$ obviously has its major impact by further reducing radiation through the compensation of lower pitch values.

Certainly, the decision on whether or not implementing the protocol with tube current reduction to $4 \%$ relies on the intent whether or not to use data for analysis of ventricular function. Whereas data at $20 \%$ of the full tube current are of adequate image quality to allow for accurate analysis of ventricular function, data at $4 \%$ are no longer useful for that purpose. Most recently, it has been shown that high quality images throughout the cardiac cycle may still be available despite the use of ECG-based tube current reduction through interpolation of data that were acquired during the normal tube current phase [22].

\section{Radiation dose in cardiac CT vs. catheter angiography}

Reviewing the literature reveals that studies on radiation exposure caused by catheter coronary angiography are conspicuously rare. Depending on the studies, effective doses ranged from 4.2 to $21.8 \mathrm{mSv}$ for diagnostic invasive procedures [23]. Coles and coworkers [24] have recently reported a mean radiation dose of $5.6 \mathrm{mSv}$ for catheter coronary angiography. On the other hand, a recent survey on diagnostic catheter angiographies has revealed effective doses higher than $100 \mathrm{mSv}$ in $2 \%$ of the diagnostic procedures [25].

Previous single-source 64-slice CT coronary angiography studies have reported estimated radiation doses of up to $21.4 \mathrm{mSv}$ without the use of the ECG-pulsing technique [3]. On the other hand, Hausleiter et al. [16] reported a mean effective dose of $14.8 \mathrm{mSv}$ for single-source 64-slice 
CT coronary angiography without and $9.4 \mathrm{mSv}$ with use of ECG-pulsing. Our study documents an estimated mean effective dose of $7.8 \mathrm{mSv}$ or $8.8 \mathrm{mSv}$ for dual-source CT coronary angiography, depending on the ECG-pulsing protocol used.

It should be always kept in mind, however, that the overall risk of an examination is a weighted summation of the risk contribution of each component of the procedure. This means that when comparing the risk of catheter coronary angiography to that of CT coronary angiography, there are risks common to both procedures, such as adverse effects to iodinated contrast media and radiation, but there are also risks unique to each examination [26]. Catheter coronary angiography, for example, implies additional risks to the patient through a major complication rate of $1.7 \%$ associated with catheterization, yielding a considerable non-radiogenic risk of mortality [26]. This must be imposed onto the potential risk associated with X-ray radiation of the technique.

\section{Study limitations}

It is well known that mathematical estimations of effective doses for CT protocols may underestimate or overestimate the true radiation exposure to the patient [27]. However, as a matter of fact, it is not possible to measure the delivered dose within a patient. Therefore, we considered in this study the most useful parameters to describe and compare radiation doses received from $\mathrm{CT}$ examinations [10]. We used a tube current-time product of $350 \mathrm{mAs}$ combined with a tube voltage of $120 \mathrm{kV}$. No studies exist so far that provide information about the lowest tube current-time product possible in order to maintain a low image noise while maintaining diagnostic image quality with dualsource CT. In addition, circumference-adapted tube current regulation [28] or tube current-time product adaptation to body weight combined with online tube current modulation [29] has been shown to deliver chest examinations with lower dose and without loss of information. Another important approach for reducing radiation dose is the reduction of tube voltage [16], because the radiation dose varies with the square of the tube voltage. In addition, we did not assess the feasibility and radiation dose of dualsource CT coronary angiography using prospective ECGtriggering, which is known to further reduce radiation. Further studies are needed to determine the lowest acceptable tube current-time product and tube voltage to maintain diagnostic image quality and to assess the feasibility of prospective ECG-triggering for CT coronary angiography.

\section{Conclusion}

Dual-source CT coronary angiography with $350 \mathrm{mAs} /$ rotation and $120 \mathrm{kV}$ results in an estimated mean effective dose of $8.8 \mathrm{mSv}$ or $7.8 \mathrm{mSv}$, depending on the ECGpulsing algorithm used. Radiation dose associated with the protocol reducing tube current to $20 \%$ outside the pulsing window significantly decreases with increasing heart rates, despite using wider pulsing windows at higher heart rates in order to maintain diagnostic image quality. Radiation dose stays constant at a significantly lower level when using ECG-pulsing with reduction of tube current to $4 \%$, irrespective of the heart rate during the examination.

Acknowledgements This research has been supported by the National Center of Competence in Research, Computer Aided and Image Guided Medical Interventions of the Swiss National Science Foundation.

\section{References}

1. Leber AW et al (2005) Quantification of obstructive and nonobstructive coronary lesions by 64-slice computed tomography: a comparative study with quantitative coronary angiography and intravascular ultrasound. J Am Coll Cardiol 46:147-154

2. Leschka S et al (2005) Accuracy of MSCT coronary angiography with 64slice technology: first experience. Eur Heart J 26:1482-1487

3. Mollet NR et al (2005) High-resolution spiral computed tomography coronary angiography in patients referred for diagnostic conventional coronary angiography. Circulation 112:2318-2323
4. Raff GL, Gallagher MJ, O’Neill WW, Goldstein JA (2005) Diagnostic accuracy of noninvasive coronary angiography using 64-slice spiral computed tomography. J Am Coll Cardiol 46:552-557

5. Flohr TG et al (2006) First performance evaluation of a dual-source CT (DSCT) system. Eur Radiol 16:256-268

6. Achenbach S et al (2006) Contrastenhanced coronary artery visualization by dual-source computed tomographyinitial experience. Eur J Radiol 57:331335

7. Johnson TR et al (2006) Dual-source CT cardiac imaging: initial experience. Eur Radiol 16:1409-1415
8. Scheffel H et al (2006) Accuracy of dual-source CT coronary angiography: First experience in a high pre-test probability population without heart rate control. Eur Radiol 16:2739-2747

9. Yates SJ, Pike LC, Goldstone KE (2004) Effect of multislice scanners on patient dose from routine CT examinations in East Anglia. Br J Radiol 77:472-478

10. Gerber TC, Kuzo RS, Morin RL (2005) Techniques and parameters for estimating radiation exposure and dose in cardiac computed tomography. Int $\mathrm{J}$ Cardiovasc Imaging 21:165-176 
11. Primak AN, McCollough $\mathrm{CH}$, Bruesewitz MR, Zhang J, Fletcher JG (2006) Relationship between noise, dose, and pitch in cardiac multidetector row CT. Radiographics 26:1785-1794

12. Jakobs TF et al (2002) Multislice helical CT of the heart with retrospective ECG gating: reduction of radiation exposure by ECG-controlled tube current modulation. Eur Radiol 12:1081-1086

13. McCollough $\mathrm{CH}$ et al (2007) Dose performance of a 64-channel dualsource CT scanner. Radiology 243:775-784

14. Leschka S et al (2007) Image Quality and Reconstruction Intervals of DualSource CT Coronary Angiography: Recommendations for ECG-Pulsing Windowing. Invest Radiol 42:543-549

15. Menzel H, Schibilla H, Teunen D, eds. (2000) European guidelines on quality criteria for computed tomography. Luxembourg: European Commission Publication No. EUR 16262 EN

16. Hausleiter J et al (2006) Radiation dose estimates from cardiac multislice computed tomography in daily practice: impact of different scanning protocols on effective dose estimates. Circulation 113:1305-1310
17. Morin RL (1988) Monte carlo simulation in the radiological sciences. CRC Press, Boca Raton, FL

18. Austen WG et al (1975) A reporting system on patients evaluated for coronary artery disease. Report of the Ad Hoc Committee for Grading of Coronary Artery Disease, Council on Cardiovascular Surgery, American Heart Association. Circulation 51:5-40

19. McCollough CH (2003) Patient dose in cardiac computed tomography. Herz $28: 1-6$

20. McCollough CH, Schueler BA (2000) Calculation of effective dose. Med Phys 27:828-837

21. Schardt P et al (2004) New x-ray tube performance in computed tomography by introducing the rotating envelope tube technology. Med Phys 31:26992706

22. Wierzbicki M, Guiraudon GM, Jones DL, Peters T (2007) Dose reduction for cardiac CT using a registration-based approach. Med Phys 34:1884-1895

23. Kuon E, Robinson DM, Empen K, Dahm JB (2005) [Fluoroscopy time an overestimated factor for patient radiation exposure in invasive cardiology]. Rofo 177:812-817

24. Coles DR et al (2006) Comparison of radiation doses from multislice computed tomography coronary angiography and conventional diagnostic angiography. J Am Coll Cardiol 47:1840-1845
25. Aroua A, Trueb P, Vader JP, Valley JF, Verdun FR (2007) Exposure of the Swiss population by radiodiagnostics: 2003 review. Health Phys 92:442-448

26. Zanzonico P, Rothenberg LN, Strauss HW (2006) Radiation exposure of computed tomography and direct intracoronary angiography: risk has its reward. J Am Coll Cardiol 47:18461849

27. Becker C et al (1999) [Assessment of the effective dose for routine protocols in conventional CT, electron beam CT and coronary angiography]. Rofo 170:99-104

28. Das M et al (2005) Individually adapted examination protocols for reduction of radiation exposure for 16MDCT chest examinations. AJR Am J Roentgenol 184:1437-1443

29. Nyman U, Ahl TL, Kristiansson M, Nilsson L, Wettemark S (2005) Patientcircumference-adapted dose regulation in body computed tomography. A practical and flexible formula. Acta Radiol 46:396-406 